\title{
Nanosatellite case study: Issue of heat dissipation across passive thermal analysis
}

\author{
Amine $\mathrm{AKKA}^{1 *}$, Farid BENABDELOUAHAB ${ }^{2}$, and Randa YERROU $^{3}$ \\ ${ }^{1,2}$ Laboratory of Condensed Matter Physics, Department of Physics, Faculty of Science at Abdelmalek Essaadi University, B, P. 2121 \\ M'Hannech II. 93030 Tetouan. Kingdom of Morocco \\ ${ }^{3}$ Radiation \& Nuclear Systems Laboratory, Department of Physics, Faculty of Science at Abdelmalek Essaadi University, B, P. 2121 \\ M'Hannech II. 93030 Tetouan. Kingdom of Morocco
}

\begin{abstract}
One of the major reasons, why nanosatellites are still in a state of vulnerability, is the increase of their thermal temperature, which in many cases leads to the failure of the missions they were originally designed for. In order to maintain the quality of the thermal management system, while keeping the electronic equipment in reliable working conditions. This paper presents a research on the thermal environment and the passive thermal control systems (PTC) of nanosatellites, based on a CFD computational fluid dynamics tool. The idea of this work is to provide a PTC to nanosatellites at a specific altitude in low earth orbit in order to determine their temperature values. From the results collected, the temperatures of the nanosatellites change drastically depending on the presence or absence of heat dissipation. This also depends on the type of the considered coating. It was also noticed that the temperature inclined with the choice of surface coating which was stipulated by the $\alpha / \varepsilon$ ratio. Therefore, it would be wise to choose the right surface coating at the very beginning of the design.
\end{abstract}

Keywords: Radiation heat transfer; nanosatellite; heat dissipation; passive thermal analysis; optical coatings;

\section{Introduction}

Orbiting spacecraft cope with the question of high thermal gradients or different thermal loads negotiated by different solar radiation. Thermal control of a spacecraft can guarantee the temperature maintenance of its various components within their appropriate ranges. Simulation and temperature prediction of a spacecraft during the flight are usually carried out using a commercial code.

Small satellite projects as "The CubeSat project" are made possible thanks to the miniaturization of components. Although the integration of compact electronic devices allows the realization of complex scientific experiments and space missions [1], thermal control options for small satellites are still limited. Indeed even if traditional temperature control techniques have confirmed their capability and effectiveness in large spacecraft, they may need to be further improved for controls and tests that revolve around small spacecraft applications [2].

Thermal insulated structural joints are one of several passive thermal control methods. Indeed, this method of achieving thermal balance of the spacecraft is mainly based on the installation of multiple low thermal conductivity washers between the connectors and the bonded surfaces in order to limit thermal conduction to specific locations [3].

In the following, the passive thermal control method used is rather the one based on the thermo-optical characteristics of the nanosatellite coatings [4-5].

\section{Nanosatellite state environment}

In vacuum, heat is transferred in two ways either by radiation or conduction. The internal environment of a small fully enclosed satellite is mainly dominated by the conductive heat flux, while the overall energy balance and the external environment are governed only by thermal radiation. The radiant heat environment is handled with materials that have specific radiation properties commonly known as solar radiation absorption (which includes wavelengths ranging from about 0.3 to $3 \mu \mathrm{m}$ ) and infrared emissivity (about 3 to $50 \mu \mathrm{m})$. The spacecraft absorption of sunlight determines the amount of penetrating solar flux, while IR emissivity determines the degree to which the spacecraft emits its thermal energy in space relative to a black body emitter. These properties are entirely those

\footnotetext{
* Corresponding author: a akka@hotmail.com
} 
of a material surface and can be modified simply by adding specialized coatings, electroplating, polishing and even adhesive tapes of particular materials.

The main reason for nanosatellite's thermal design limitation is that the passive thermal control subsystem is generally developed considering restricted weight and power budgets. However, other constraints also affect the design of the thermal control subsystem of this kind of satellites [6].

The influence of various factors, including the emission characteristics, thickness, structures and processing techniques of thermal parameters, was evaluated experimentally and theoretically [7].

This paper focuses on the thermal study and analysis of a nanosatellite designed for a circular low earth orbit. It has a spherical form geometry with a radius equivalent to a cube of $10 \mathrm{~cm} \times 10 \mathrm{~cm} \times 10 \mathrm{~cm}$ measurements. A passive thermal control was designed according to the mission requirements. Different aspects of surface coatings were used to accomplish the required thermal control. The objective of this study is to keep the overall temperature of the nanosatellite within the proper limits and to provide appropriate measures to ensure the operation of all components within their safe temperature range with proper denial.

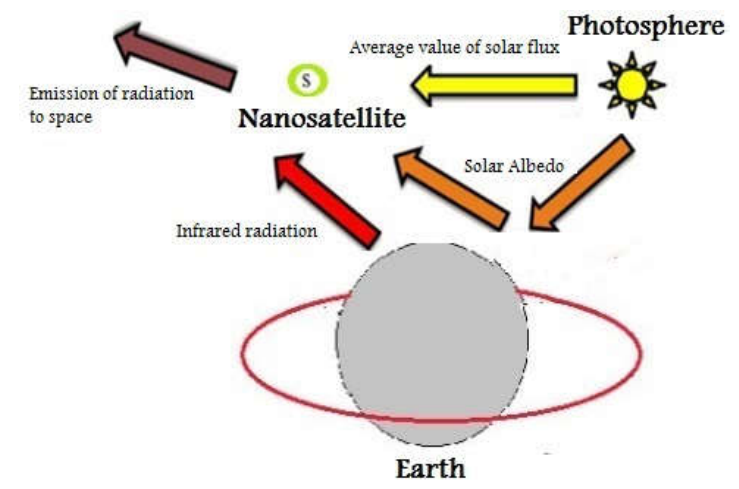

Fig 1. Nanosatellite Energy Balance

Thermal control is an energy management process in which space environmental heating plays a major role. The main forms of heating of the orbiting environment are direct sunlight (Photosphere), ground-reflected sunlight (albedo) and Earth infrared radiation (IR) emitted by the Earth. The amount of external heat absorbed directly namely solar energy depends on the material properties of the nanosatellite and its orientation to the Sun. As it can be seen in Figure 1, the heat balance between the nanosatellite and the space environment involves many parameters. The temperature of the nanosatellite is the outcome of the balance between the energy absorbed and the energy emitted by all of these sources.

\section{Nanosatellite thermal design}

The purpose of this paper is to focus on the thermal design and results of the detailed steady-state thermal analysis of a spherical nanosatellite at a specific altitude with a combination of optical coatings each with specific properties namely the infrared emissivity and the solar absorptivity [8]. The thermal control system ensures that all components are operating within the temperature limits allowed for the whole mission of the satellite, especially batteries which still a weak link regarding their flimsy margin of temperature change. All components must function from the start to the end of their life-cycle under the required performances. Thermal control can be passive or active, depending on the design approach.

This work extends several previous research in the challenging field of nanosatellite thermal simulation, and the results achieved are discussed with reference to other recent, relevant and innovative works $[9,10,11]$.

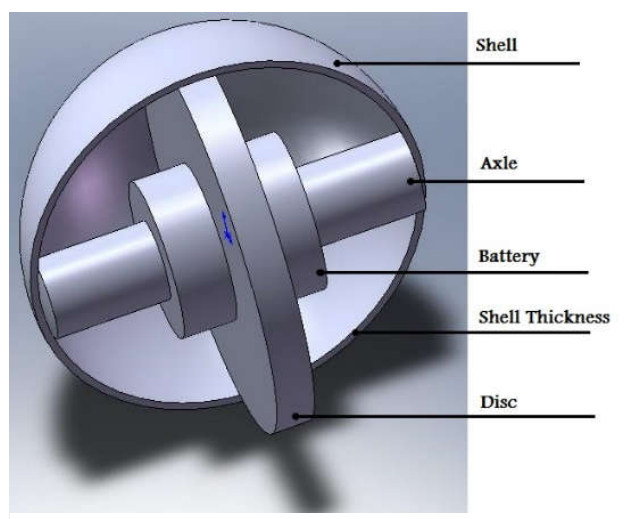

Fig 2. Nanosatellite structure

The nanosatellite presented in Figure 2 has a structure mainly made of aluminium alloy. In this fundamental model, the shape considered is spherical with a disk mounted in the middle supposed to contain the electronic components whose energy dissipation is estimated to be not very important compared to that of the batteries. The latter are attached on one side to the disk and on the other to two axes which ensure the stability of the nanosatellite when it is in orbit. In order to create a sufficient thermal barrier and to allow a high thermal resistance, a thickness of two millimeters was required at the shell's level [12].

Table 1 presents the measurements of solar flux, terrestrial albedo and terrestrial IR values [13]. For brevity, the orbit is assumed to be circular and the contribution of the aerothermal flux is omitted due to altitude. Let us further assume that the nanosatellite has a high thermal inertia, due to its isothermal surface.

Table 1. Input variables.

\begin{tabular}{|l|c|}
\hline \multicolumn{1}{|c|}{ Parameters } & Value \\
\hline Solar radiation heat flux $\left(\mathrm{W} / \mathrm{m}^{2}\right)$ & 1371 \\
\hline Earth radiation heat flux $\left(\mathrm{W} / \mathrm{m}^{2}\right)$ & 220 \\
\hline Albedo factor & 0.33 \\
\hline Orbital height $(\mathrm{Km})$ & 240 \\
\hline Temperature \& pressure & Vacuum at $2.7^{\circ} \mathrm{K}$ \\
\hline Average Heat dissipation $(\mathrm{W})$ & 5 \\
\hline
\end{tabular}


Table 2 shows the properties of the surface materials [14]. A more detailed description of the material properties can be found by referring to [15].

It is obvious that the main objective of thermal management is to keep the nanosatellite thermal energy in balance, yet also to ensure that all of the internal components stay within their acceptable temperature limits during the entire space journey.

As mentioned earlier, the analysis developed in this work was entirely devoted to a steady-state analysis that plots the distribution of the spacecraft's temperature and all of its components under steadystate loading conditions. This type of analysis is defined as a statement where heat storage over a period of time is neglected.

Table 2. Coating properties

\begin{tabular}{|c|c|c|c|}
\hline $\begin{array}{c}\text { Coating } \\
\text { designation }\end{array}$ & $\begin{array}{c}\text { Absorptance } \\
\boldsymbol{\alpha}\end{array}$ & $\begin{array}{c}\text { Emissivity } \\
\boldsymbol{\varepsilon}\end{array}$ & $\begin{array}{c}\text { Ratio } \\
\boldsymbol{\alpha} / \boldsymbol{\varepsilon}\end{array}$ \\
\hline White paint & 0.2 & 0.88 & 0.23 \\
\hline $\begin{array}{c}\text { Black paint } \\
\text { (Electrodrag } \\
\text { 501) }\end{array}$ & 0.94 & 0.81 & 1.16 \\
\hline VDA & 0.15 & 0.05 & 3 \\
\hline VDAu & 0.23 & 0.025 & 9.20 \\
\hline
\end{tabular}

The basic energy balance, once the equilibrium is reached, used for the calculation of steady-state temperatures is commonly stated as:

$$
Q_{\text {in }}=Q_{\text {out }}
$$

Solving the energy balance equation is the essential aim of the thermal analysis that is summed up by resolving the Eq. (1) above, and also by finding the spatial temperature distribution, and its temporal evolution.

In each nano-domain, the distribution of the temperature can be yielded analytically or numerically by coded solvers.

At a given time, the heat balance of the ith node of a material made up of $n$ nodes is given below Eq. (2). Note that the thermal modeling relies on splitting the simpler geometry system into several subdomains so that adjacent surfaces of the nanosatellite do not interfere with each other, ensuring that the reliability and accuracy of the temperature result are not compromised.

$$
\begin{gathered}
\sum_{j=1}^{n} L_{i j} \cdot\left(T_{i}-T_{j}\right)+\sum_{j=1}^{n} A_{i} \cdot F_{i j} \cdot \varepsilon_{i j} \cdot \sigma \cdot\left(T_{i}^{4}-T_{j}^{4}\right)+\left(m_{i} \cdot C_{i}\right) \cdot \frac{d T_{i}}{d t}=Q_{s u, i} \\
+Q_{a, i}+Q_{e, i}+Q_{d, i}-Q_{s a t, i}
\end{gathered}
$$

- The analysis is limited to the steady-state, so $\mathrm{dT}_{\mathrm{i}} / \mathrm{dt}=0$.

- $\mathrm{L}_{\mathrm{ij}}$, the effective conductance between nodes $\mathrm{i}$ and $\mathrm{j}$.

- $\mathrm{T}_{\mathrm{i}}$ and $\mathrm{T}_{\mathrm{j}}$, the temperatures of the ith and jth nodes.

- $\mathrm{A}_{\mathrm{i}}$, the area of surface $\mathrm{i}$.

- $F_{i j}$, the view factor of surface $\mathrm{j}$ as seen from surface

- $\varepsilon_{\mathrm{ij}}$, the effective emittance, where $\varepsilon_{i j}=\frac{\varepsilon_{i} \varepsilon_{j}}{\varepsilon_{i}+\varepsilon_{j}-\varepsilon_{i} \varepsilon_{j}}$ emittance of surfaces $\mathrm{i}$ and $\mathrm{j}$ radiating in the infrared region.

\section{- $\sigma=5.67 \times 10^{-8} \mathrm{~W} \cdot \mathrm{m}^{-2} \cdot \mathrm{K}^{-4}$, Stefan-Boltzmann} constant.

Where, in the left side of Eq. (2), the heat conducted from the ith to the jth node is given by,

$$
L_{i j}\left(T_{i}-T_{j}\right)
$$

And the amount of radiation leaving a surface $i$ and absorbed by a surface $\mathrm{j}$ can be of the form,

$$
A_{i} F_{i j} \varepsilon_{i j} \sigma\left(T_{i}^{4}-T_{j}^{4}\right)
$$

Therefore, in the right side of Eq. (2), the solar heat received directly from the Sun may be written as follows,

$$
Q_{\text {sun }, i}=J_{s} \cdot \alpha_{i} \cdot A_{\text {sun }, i}
$$

Where $A_{\text {sun, } i}$ is the projected area, and $\mathrm{J}_{\mathrm{s}}$ is the solar constant.

Albedo contribution is noted as follows,

$$
Q_{a, i}=J_{a} \cdot \alpha_{i} \cdot A_{a, i}
$$

Where $\mathrm{A}_{\mathrm{a}, \mathrm{i}}$ is the projected area, and $\mathrm{J}_{\mathrm{a}}$ is the intensity of the albedo radiation.

For Earth radiation contribution, heat is noted as follows,

$$
Q_{e, i}=J_{e} \varepsilon_{i} A_{e, i}
$$

Where $A_{e, i}$ is the projected area, and $\mathrm{J}_{\mathrm{e}}$ is the intensity of the planetary infrared radiation.

Heat radiated to space is noted,

$$
Q_{\text {sat }, i}=\sigma \cdot \varepsilon_{i} \cdot A_{\text {sat }, i} \cdot T_{i}^{4}
$$

Where $\mathrm{A}_{\text {sat,i }}$ is the projected area, $\mathrm{T}_{\mathrm{i}}{ }^{4}$ is the temperature to the 4 th power of node $i$.

Lastly, the heat dissipated $\mathrm{Q}_{\mathrm{d}, \mathrm{i}}$ was approximately considered as that one of batteries due to their high energy dissipation in regard to the rest of components.

It is certain that a number of factors shape the external heat loads of nanosatellites. The most significant of which are specific orbit, satellite orientation and surface properties. It is also important to consider that the spinning effect of the satellite when orbiting can cause the receipt of heat in one side and dissipate it through the remaining sides especially when the nanosatellite has a prismatic shape.

Meshing remains one of the most important steps in performing a simulation, because if that task is misapplied, there would be a great chance of missing the results and stepping over the anomalies that should be avoided in the risky areas. It was therefore wise to perform a mesh based on an automatic algorithm, after which the parameter settings were rigorously executed. Indeed, the physical preference of the mesh was specified as mechanical, while for complex areas the mesh was set to tetrahedral in order to better fit with the most challenging geometric patterns. The mesh program was refined in areas with large curvatures since physical phenomenon generally tend to develop there, however the mesh remained fine for the rest of the nanosatellite.

The orthogonal quality metric was used to evaluate the quality of the mesh, which, according to the simulation, was closer to unity, ensuring thereby a good mesh quality. It was also very important to keep the computation time as short as possible while minimizing the number of available meshes. 


\section{Results and discussion}

It was appropriate to present the results with a case differentiation concerning heat dissipation or not through a computational fluid dynamics tool based on a finite element code which's the capability to obtain the evolution with time of the temperature's distribution [16-17]. In this section, the results presented were defined assuming that the dissipated energy encompassed the entire nanosatellite's body without specifying the element responsible for this dissipation.

The results obtained were validated by comparing them with those achieved by P. Poinas [14] at the European Space Agency Centre for almost the same ratios $\alpha / \varepsilon$.

The detailed thermal analysis results for the chosen data and the boundary conditions are laid out as follows,

As it can be seen in Figure 3 below, with the orbital conditions chosen and discussed above, in addition to the ratio of the material-dependent optical coating $\alpha / \varepsilon=0.23$. It is clear that the temperature of the spacecraft has increased considerably for $5 \mathrm{~W}$ of dissipated energy and has allowed the nanosatellite to jump from $-50{ }^{\circ} \mathrm{C}$ which places the structure of the satellite outside its limits of space temperature, to $-18^{\circ} \mathrm{C}$. This temperature value remains widely accepted by the structure and within the temperature limits supported by the batteries.

It should be noted that the temperature difference between the dissipation case and the case with no dissipation for this type of coating reached $32{ }^{\circ} \mathrm{C}$.

With regard to the directional flux in Figure 4 along the $\mathrm{X}$ axis, it is noted that it is not as important and that the difference between the minimum and maximum values is very insignificant.

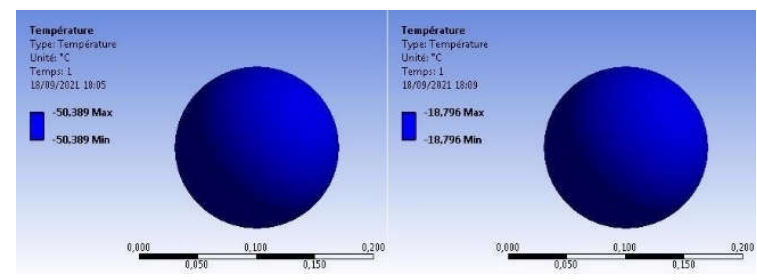

Fig 3. Temperature at ratio $\alpha / \varepsilon=0.23$; in the left: No heat dissipation, in the right: Heat dissipation.

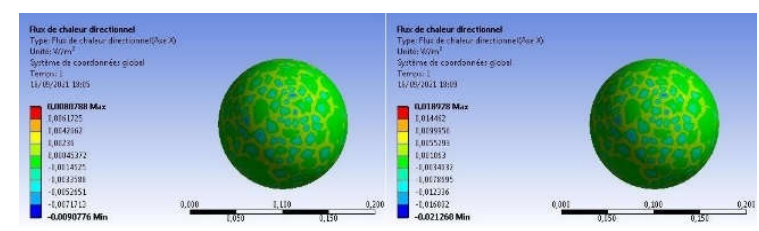

Fig 4. Axial heat flux at ratio $\alpha / \varepsilon=0.23$; in the left: No heat dissipation, in the right: Heat dissipation.

In Figure 5 below, it is clear that the impact of the ratio $\alpha / \varepsilon=1.16$ did have a significant influence on the overall temperature of the nanosatellite. It reached $34{ }^{\circ} \mathrm{C}$ without energy dissipation and $48{ }^{\circ} \mathrm{C}$ with energy dissipation. These values are supported by the structure and by the batteries.
However, it does point out that when the $\alpha / \varepsilon$ ratio is close to the unity, similar to a black body, one approaches an average temperature around $20^{\circ} \mathrm{C}$.

Note in this case that the temperature difference is $12{ }^{\circ} \mathrm{C}$ between the case with energy dissipation and the case where there is no dissipation. This suggests that once the ratio $\alpha / \varepsilon$ get close to the unity, the temperature difference decreases.

For the directional heat flux in Figure 6, the ratio change did not influence its distribution that much throughout the structure of the nanosatellite, in addition, the difference between the maximum and minimum value remained very small.

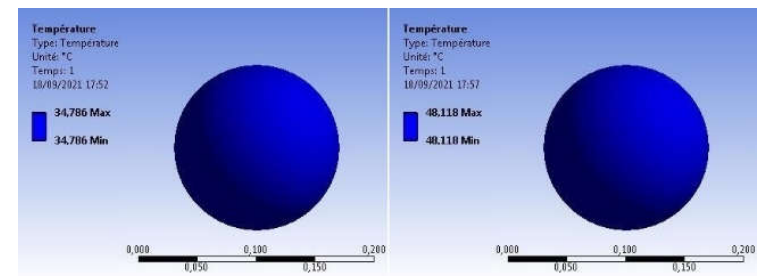

Fig 5. Temperature at ratio $\alpha / \varepsilon=1.16$; in the left: No heat dissipation, in the right: Heat dissipation.

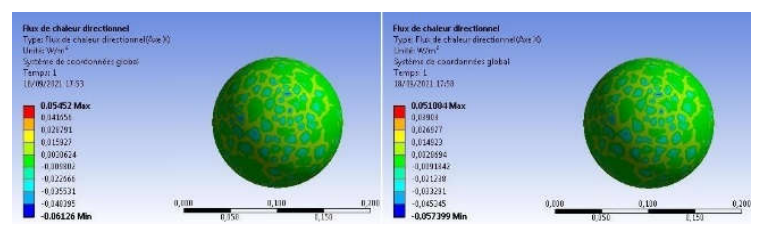

Fig 6. Axial heat flux at ratio $\alpha / \varepsilon=1.16$; in the left: No heat dissipation, in the right: Heat dissipation.

In Figure 7 below, we can clearly see that the ratio $\alpha / \varepsilon=3$ did well to increase the nanosatellite's temperature, whether it was in the case of energy dissipation, reaching $202^{\circ} \mathrm{C}$, or when there is no energy dissipation, hitting $105{ }^{\circ} \mathrm{C}$. Those temperature values exceeded the supported temperature of the structure and that of batteries and even that of photovoltaic panels if they exist.

In this case, it is clear that an external coating with this type of material must be avoided and that a thermal re-analysis must be carried out.

It is clear also that the temperature difference between the dissipation case and the case with no dissipation for this type of coating hit $97^{\circ} \mathrm{C}$.

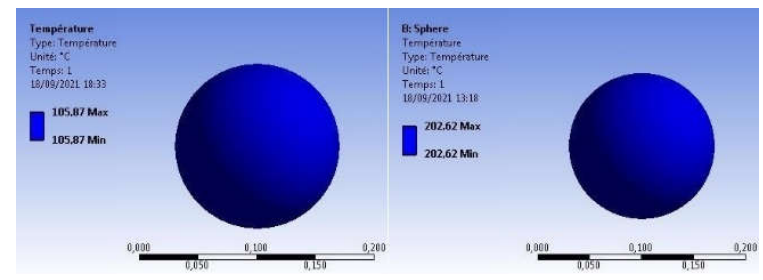

Fig 7. Temperature at ratio $\alpha / \varepsilon=3$; in the left: No heat dissipation, in the right: Heat dissipation. 
With regard to the axial heat flux in Figure 8, it is very apparent that the difference between the maximum and minimum limits remains very low but it is noted that in the case of heat dissipation, the value of the flux is very weak.

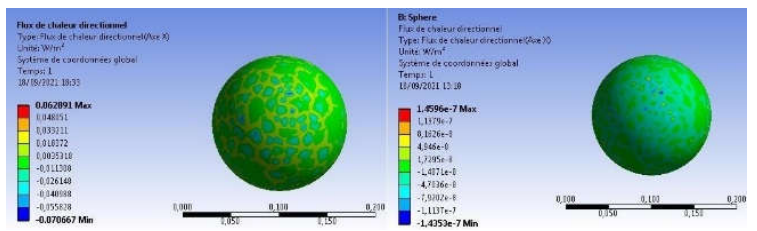

Fig 8. Axial heat flux at ratio $\alpha / \varepsilon=3$; in the left: No heat dissipation, in the right: Heat dissipation.

In Figure 9 below, we can definitely see that the temperatures reached are enormous, including $221{ }^{\circ} \mathrm{C}$ in the case where there is no energy dissipation and $312^{\circ} \mathrm{C}$ when there has been energy dissipation. However, the type of coating used is not adequate for the nanosatellite components to withstand the conditions encountered in orbit.

Here again, we could see that the difference between the case where there is energy dissipation and the case where there is no energy dissipation was close to $91{ }^{\circ} \mathrm{C}$.

In Figure 10, the axial heat flux remains with no huge change in terms of values and the limits reached are practically small so that one cannot notice its impact on the structure.

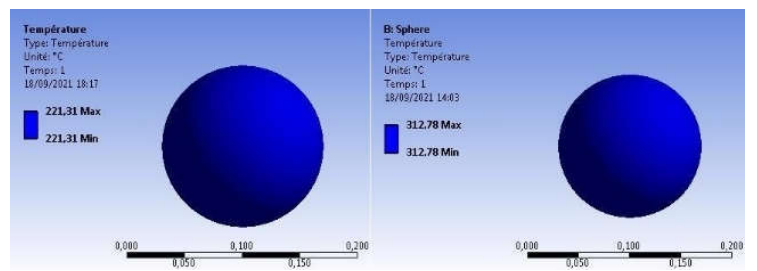

Fig 9. Temperature at ratio $\alpha / \varepsilon=9.2$; in the left: No heat dissipation, in the right: Heat dissipation.

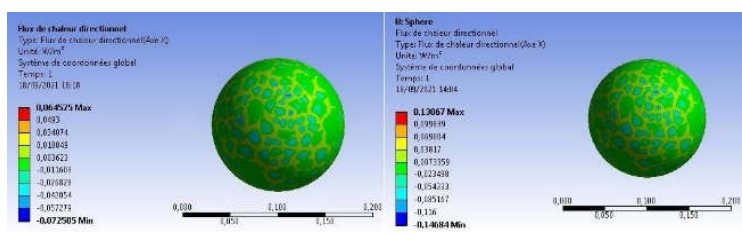

Fig 10. Axial heat flux at ratio $\alpha / \varepsilon=9.2$; in the left: No heat dissipation, in the right: Heat dissipation.

\section{Conclusion}

In this paper, the primary objective was to focus on the thermal design and detailed steady-state thermal analysis results of a nanosatellite at low earth altitude with selected optical coatings. For this purpose, a spherical shaped nanosatellite was briefly presented with all its necessary components.

Then the question of energy dissipation was treated for several types of thermo-optical coating. It was noticed that the obtained results were convincing especially that the energy dissipation was not in any case specific to a very precise element, namely the batteries.
It was also noticed that the temperature of the nanosatellite reached an acceptable rate, in particular when the ratio of the optical coating was close to the unity. This clearly shows the impact of this parameter on the temperature of the spacecraft, especially when it is about passive thermal control.

It must be said that optical coatings and the choice of adequate coating materials help much dealing with temperature variations, yet still that there are other ways to affect the temperature of a nanosatellite likely by acting on heat pipes or even by using insulation systems. Those last may be the subject of the upcoming research articles.

\section{References}

1. Y.-K. Park, G.-N. Kim, et S.-Y. Park, « Novel Structure and Thermal Design and Analysis for CubeSats in Formation Flying », Aerospace, vol. 8, no 6, p. 150, 2021.

2. R. Oliver, B. G. Crawford, et G. Burrow, « Optimized undergraduate thermal analysis of cube satellites », in 13th International Energy Conversion Engineering Conference, 2015, p. 3986.

3. C. D'Souza and R. Zanetti, «A First Look at the Navigation Design and Analysis for the Orion Exploration Mission $2 », 2017$.

4. A. Akka and F. Benabdelouahab, «PASSIVE THERMAL ANALYSIS OF A CUBESAT BY A FINITE ELEMENT MODELING », JPHMT, vol. 21, no 1, p. 133-149, Oct. 2020, doi: 10.17654/HM021010133.

5. A. Akka and F. Benabdelouahab, "Nanosatellite: A Progressive Vision of Performing Passive Thermal Control". in AIP Conference Proceedings, AIP Publishing LLC, vol. 2399, (In Press).

6. J. Rotteveel and A. R. Bonnema, « Thermal control issues for nano-and picosatellites $»$, in 57 th International Astronautical Congress, 2005, p. B5-6.

7. C. Shengzhu, C. Xuekang, W. Xiyuan, H. Chuang, and Y. Jianping, « Novel type of micro-variable radiator for spacecraft thermal control », Chin. J. Vac. Sci. Technol, vol. 8, 2013.

8. V. L. Pisacane, Fundamentals of space systems. Johns Hopkins University/Appli, 2005.

9. M. Bulut, Ö. R. Sözbir, and N. Sözbir, « Thermal Control of Turksat 3U Nanosatellite », 2017.

10. A. Akka, F. Benabdelouahab and R. Yerrou, EVALUATING THE TEMPERATURE TOGGLING OF A NANOSATELLITE THRU A PRELIMINARY PASSIVE THERMAL ANALYSIS, JP Journal of Heat and Mass Transfer 24(2), (In Press). 
11. G. Salazar-Salinas, E. Botello-Ramírez, and

E. Avalos-Gauna, « Thermal Analysis of a 3U-

Cubesat, a Case Study of Pakal Satellite »,

presented at The International Conference on Fluid

Flow, Heat and Mass Transfer (FFHMT'21),

Virtual Conference, May 2021. doi:

10.11159/ffhmt21.137.

12. P. Shinde, A. Quintero, I. Tansel, et S. Tosunoglu, «CubeSat Thermal Analysis », presented at 30th Florida Conference on Recent Advances in Robotics, Florida Atlantic University, Boca Raton, Florida, May 2017.

13. M. Donabedian and D. G. Gilmore, « Spacecraft thermal control handbook », Aerospace Press, p. 21-69, 2003.

14. Philippe. Poinas, « Satellite Thermal Control Engineering », in SME04, ESTEC Thermal \& Structure Division, June 2004, p. 66.

15. Specialists in materials and applications, «Spacecraft Thermal Control and Conductive Paints/Coatings and Services Catalog ». AZ Technology, 2008, [On line]. Available at: http://www.aztechnology.com.

16. B. Končar and L. Klobučar, « Use of CFD Codes for Calculation of Radiation Heat Transfer: From Validation to Application », Heat TransferModels, Methods and Applications, Intech Open, 2018.

17. P. Vueghs, H. P. De Koning, O. Pin, and P. Beckers, «Use of geometry in finite element thermal radiation combined with ray tracing », Journal of computational and applied mathematics, vol. 234, no 7, p. 2319-2326, 2010. 